\title{
Magnetization reversal via symmetric rotation of layers in exchange biased multilayers
}

Amitesh Paul, Matthias Buchmeier, Claus M. Schneider, and Thomas Brückel

Citation: Journal of Applied Physics 101, 123913 (2007);

View online: https://doi.org/10.1063/1.2747539

View Table of Contents: http://aip.scitation.org/toc/jap/101/12

Published by the American Institute of Physics

\section{Articles you may be interested in}

Spatially resolved observation of uniform precession modes in spin-valve systems

Journal of Applied Physics 109, 07 D305 (2011); 10.1063/1.3535439

Magnetic properties of EuS spin filter tunnel contacts to silicon

Journal of Applied Physics 109, 07 C710 (2011); 10.1063/1.3549609

Normal and inverse current-induced magnetization switching in a single nanopillar

Applied Physics Letters 89, 222511 (2006); 10.1063/1.2398923

Mössbauer transmission and back scattered conversion electron study of Fe nanowires encapsulated in multiwalled carbon nanotubes

Journal of Applied Physics 96, 7514 (2004); 10.1063/1.1811781

Surface analysis of the Heusler Ni49.7 $\mathrm{Mn}_{29.1} \mathrm{Ga}_{21.2}$ Alloy: The composition, phase transition, and twinned microstructure of martensite

Journal of Applied Physics 120, 113905 (2016); 10.1063/1.4962648

Interface-mediated ferroelectric patterning and $\mathrm{Mn}$ valency in nano-structured $\mathrm{PbTiO}_{3} / \mathrm{La}_{0.7} \mathrm{Sr}_{0.3} \mathrm{MnO}_{3}$

Journal of Applied Physics 120, 095304 (2016); 10.1063/1.4962007

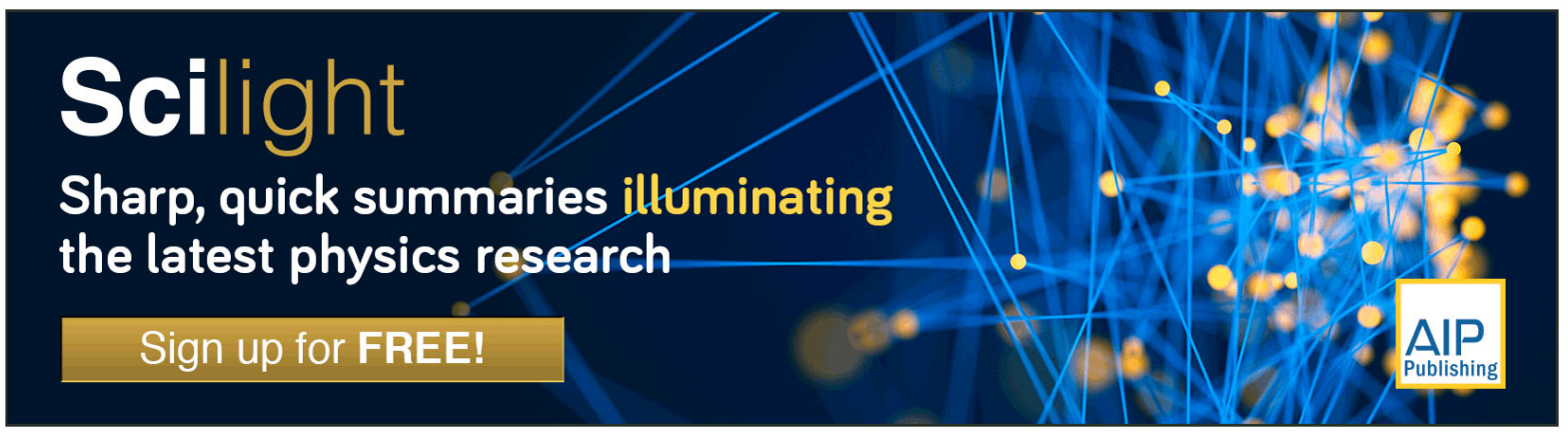




\title{
Magnetization reversal via symmetric rotation of layers in exchange biased multilayers
}

\author{
Amitesh Paul, ${ }^{\text {a) }}$ Matthias Buchmeier, Claus M. Schneider, and Thomas Brückel \\ Institut für Festkörperforschung, Forschungszentrum Jülich GmbH, D-52425 Jülich, Germany
}

(Received 11 April 2007; accepted 16 April 2007; published online 25 June 2007)

\begin{abstract}
We have investigated the magnetization reversal for exchange coupled polycrystalline $[\mathrm{IrMn} / \mathrm{CoFe}]_{N}$ multilayers. Polarized neutron reflectivity (PNR) data indicate a simultaneous coherent rotation of all ferromagnetic layers for a sample with $N=10$ and angles of $45^{\circ}$ and $90^{\circ}$ between the applied field and the exchange bias direction. On the other hand, magneto-optic Kerr effect (MOKE) measurements, which are sensitive mainly to the two topmost bilayers, reveal a variation of the strength of the exchange bias and the uniaxial anisotropy as a function of $N$ for multilayers with $N=1$ up to 10 . The MOKE data thus indicate the direction of the magnetization to vary from layer to layer for intermediate fields. PNR was found to be insensitive to this variation as the deviation of the layer magnetization directions from its mean value is relatively small $\left(\approx 10^{\circ}\right)$. These studies demonstrate how the complementary techniques PNR and MOKE can be used to obtain a layer-by-layer vector magnetometry of multilayer stacks. (C) 2007 American Institute of Physics. [DOI: 10.1063/1.2747539]
\end{abstract}

\section{INTRODUCTION}

Exchange biased spin valves have been established as an integral part of magnetic recording devices, in particular, in read heads. When a ferromagnetic (FM) layer is brought into direct contact with an antiferromagnetic (AF) layer and is cooled below the Néel temperature then a shift in the hysteresis loop of this bilayer structure is observed. This shift on the magnetic field axis, which is referred to as the exchange bias field, is crucial for designing thin film magnetic sensors in recording devices. However, understanding the basic mechanism of the exchange bias phenomena itself is still elusive even half a century after its discovery. ${ }^{1}$

As the exchange-biasing mechanism introduces a unidirectional magnetic anisotropy in the ferromagnetic layer, the magnetization reversal process of such systems is quite unusual, when compared to that of any single uncoupled ferromagnetic layer, which can at best exhibit a uniaxial anisotropy. Thus, an asymmetric reversal behavior is frequently observed in exchange coupled ferromagnet/ antiferromagnetic systems. ${ }^{2-6}$ This asymmetry appears between the increasing and decreasing (with respect to the cooling field direction $H_{\mathrm{FC}}$ ) field branches of the hysteresis. The magnetization reversal has been found to be symmetric, when both branches of the hysteresis, increasing as well as decreasing $H_{a}$, are governed by the same microscopic reversal mechanism, which can involve either a uniform mode (by coherent rotation) or a nonuniform mode (reversal by domain nucleation and wall motion). On the other hand, the reversal tends to be asymmetric, when the microscopic modes are different for both branches. The magnetization reversal is basically governed by an effective field $H_{\text {eff }}$ arising from the anisotropy of the FM, the exchange bias field $H_{X}$ due to the AF, and the applied field $H_{a} . H_{\text {eff }}$ and the

\footnotetext{
${ }^{a)}$ Author to whom correspondence should be addressed; electronic mail: a.paul@fz-juelich.de
}

torque that it exerts on the FM magnetization depend on the angle $\theta$ between the exchange bias and applied field directions. The theoretical interpretation of the magnetization reversal has been discussed in detail by Beckmann et al. ${ }^{7}$ It has been shown that depending on $\theta$ the hysteresis can be either symmetric or asymmetric.

Recently, Paul et al. ${ }^{8}$ observed the strength of the exchange bias field to increase when FM and AF layers are repeated for a number of times $N$ forming a multilayer (ML). An increase of the strength of the bias field with $N$ was directly related to the evolution of the grain size along the stack. It can be attributed to a higher density of uncompensated spins, which has its origin in the domain state of the AF stabilized by nonmagnetic defects such as grain boundaries.

This evolution of the bias field along the stack has eventually lead to the observation of a sequential reversal of the FM layers in polycrystalline $[\mathrm{IrMn} / \mathrm{CoFe}]_{10}$ (Refs. 8 and 10) and also in $[\mathrm{Co} / \mathrm{CoO}]_{20}$ MLs (Ref. 9) using polarized neutron reflectivity (PNR). Unlike the usually observed asymmetric reversal processes in epitaxial bilayer specimens for $\theta=0^{\circ}$ (Refs. 4-6 and 9), the reversal mode was found to be symmetric and proceeded via a nonuniform mode. ${ }^{8,9}$ Further, for $\theta \neq 0^{\circ}$, the relative strengths of the uniaxial and the exchange bias anisotropies in the systems could be varied within a single multilayered system, which is characterized by different strengths of the bias fields for increasing $N$ along the stack. This anisotropy variation was instrumental in deciding upon the magnetization reversal mode for each layer in the $N=10 \mathrm{ML}$ stack for $\theta \approx 15^{\circ}$ (Ref. 10). Unlike at $\theta$ $=0^{\circ}$, the reversal was found to proceed either via a uniform or a nonuniform mode, depending upon the position of the respective layer in the ML stack.

In the present paper, we investigate the details of the reversal mechanism in similar polycrystalline $\mathrm{IrMn} / \mathrm{CoFe}$ samples by combining complementary analysis techniques. The multilayers are prepared by dc magnetron sputtering and 
have the sequence $\left[\operatorname{Ir}_{20} \mathrm{Mn}_{80}(6.0 \mathrm{~nm}) / \mathrm{Co}_{80} \mathrm{Fe}_{20}(3.0 \mathrm{~nm})\right]_{N}$, with $N=1,2,3,5$, and $10 .^{8}$ A buffer layer of $\mathrm{NiFe}(10.0 \mathrm{~nm})$ was used and was found to be almost magnetically decoupled from the actual multilayer stack. In the following we present magneto-optic Kerr effect (MOKE) measurements of the longitudinal and transverse components of magnetization. The measurements are performed for different angles $(\theta)$, as mentioned above, and are compared to a single domain model with effective exchange bias. The information obtained from the MOKE data is compared with the results from new PNR measurements and earlier investigations ${ }^{8-11}$ on a $N=10$ bilayer specimen for two different directions $\theta$ $=45^{\circ}$ and $90^{\circ}$.

\section{EXPERIMENTAL DETAILS}

Both experimental techniques-MOKE and PNR - are sensitive to the in-plane magnetization for a homogeneous film. We define the longitudinal magnetization component as parallel to the applied field direction $M \cos \phi_{A}$ and the transversal magnetization component as perpendicular to the applied field direction $M \sin \phi_{A}$, where $\phi_{A}$ is the angle between the magnetization $M$ and the applied field $H_{a}$. Neutron scattering with polarization analysis can also discriminate the longitudinal and transverse components of the magnetization. The non-spin-flip (NSF) channels in specular scattering are sensitive to the longitudinal magnetization component, while the SF channels are sensitive to the transverse component. One may note that the SF reflectivities from PNR are generally proportional to the mean square of the in-plane magnetization component $\perp$ to the polarization direction or the applied field direction, i.e., $\left\langle\sin ^{2} \phi_{A}\right\rangle$, while the NSF reflectivities are proportional to the mean value of in-plane magnetization component $\|$ to the polarization axis $\left\langle\cos \phi_{A}\right\rangle$. As the sign of the magnetization is encoded in the phase of the SF reflectivities, we can only measure the modulus of $M_{\perp}$. Thus, there exists a similarity between the measured components of the magnetization from PNR and that of MOKE, and they can be compared. The main difference of the techniques lies in the information depth, which is limited in MOKE to about $100 \AA$.

\section{A. Longitudinal and transverse MOKE}

The MOKE measurements are performed using a laser light with a wavelength of $670 \mathrm{~nm}$ at an angle of incidence of $15^{\circ}$. The magnetic field generated by an electromagnet takes values in the range of $0-0.5 \mathrm{~T}$. The setup provides two light beams: a longitudinal beam with the plane of incidence parallel to the external field direction and a transversal beam with the plane of incidence perpendicular to the external field direction. The incident light is polarized in $p$ direction (with electric field $E$ parallel to the plane of incidence) employing a Glan-Taylor polarizer. The phase of the reflected light is modulated at a frequency $f=50 \mathrm{kHz}$ by means of a photoelastic modulator (PEM), with the modulation axis turned by $45^{\circ}$ relative to the $p$ direction. The light subsequently passes through a Glan-Taylor polarizer aligned in $s$ direction and is converted into an electrical signal with a diode detector. The small $2 f$ component of the signal is determined with a lock-in amplifier, whereas the much larger $f$ component is measured with a multimeter. Within first order approximation, the $2 f$ component is proportional to the $p$ ellipticity $\varepsilon_{p}\left(V_{2 f} \propto I \varepsilon_{p}\right)$ while the large $f$ component is proportional to the intensity of the reflected light $\left(V_{f} \propto I\right)$. The ellipticity has been determined by dividing the $2 f$ and $f$ components $\varepsilon$ $\propto V_{2 f} / V_{f}$. It has been calibrated by comparing the experimental ellipticity of a thick Au film proportional to a small rotation of the polarizer to its theoretical value using the index of refraction $\eta_{\mathrm{Au}}=(0.10+3.65 i)$. A schematic of the MOKE setup is given in Fig. 1.

\section{B. Neutron scattering}

The neutron scattering technique with polarization analysis has been already used to study various combinations of AF-FM bilayers. ${ }^{3-6,8-12}$ The specular reflectivities in the NSF channels $\left(R_{++}\right.$and $\left.R_{--}\right)$are due to periodicities of the structure and magnetization components collinear to $H_{a}$, whereas reflectivities in the SF channels $\left(R_{+-}\right.$and $\left.R_{-+}\right)$are exclusively of magnetic origin and correspond to in-plane magnetization components perpendicular to the guiding field $H_{a}$. In specular geometry (angle of incidence $\alpha_{i}$ equal to the exit angle $\alpha_{f}$ ), the reflectivities follow from energy and inplane momentum conservation laws as normal wave vector transfers $Q_{\perp}=(2 \pi / \lambda)\left[\sin \left(\alpha_{i}\right)+\sin \left(\alpha_{f}\right)\right]$ are probed. Offspecular scattering contributions along the in-plane momentum transfer vector $Q_{\|}=(2 \pi / \lambda)\left[\cos \left(\alpha_{f}\right)-\cos \left(\alpha_{i}\right)\right]$ arise when the in-plane translational symmetry is broken by interface roughness or by magnetic domains on a length scale shorter than the in-plane projection of the neutron coherence length $l_{\|}$(Refs. 13 and 14) along $Q_{\|}$. For our instrument $l_{\|}$exceeds $20 \mu \mathrm{m}$, but the resolution of the two-dimensional (2D) detector defines an upper limit of about 5-10 $\mu \mathrm{m}$ for the resolvable lateral structure size. ${ }^{11}$ A lower limit of about $1 \mu \mathrm{m}$ results from the limited neutron flux at the sample position. ${ }^{12}$ One should note that a magnetization reversal via rotation is identified by a significant increase of the specular reflectivities in the SF channels, which map the in-plane magnetization components perpendicular to the guiding field $H_{a}$ applied collinear to $H_{\mathrm{FC}}$. On the other hand, a reversal by domain nucleation and propagation does not lead to enhanced SF reflectivities, because the magnetization is always collinear to $H_{a}$.

The neutron scattering experiments were performed at the polarized neutron reflectometer with the polarization analysis HADAS at the Jülich research reactor FRJ-2 (DIDO). We briefly describe the configuration of HADAS that is used to determine the reflectivity. The momentum transfer vector $Q$ is given by $4 \pi \sin (\alpha) / \lambda$ when $\sin \left(\alpha_{i}\right)$ $=\sin \left(\alpha_{f}\right)$ (incident angle) $=\sin \alpha$ (exit angle) in the specular geometry. The uncertainty in $Q$, which determines the resolution, is given by $\Delta Q / Q$. For small angles $\alpha$, we have $\sin \alpha \approx \alpha$ and $\cos \alpha \approx 1$. Thus, the fraction $(\Delta Q / Q)$ can be approximated as $\sqrt{[(\Delta \lambda / \lambda) \alpha]^{2}+(\Delta \alpha / \alpha)^{2}}$. The employed graphite double monochromators provide a typical wavelength resolution of $\Delta \lambda / \lambda=1.5 \%$. For a wavelength of $4.52 \AA$, the maximum value of $\alpha$, for which one can get sufficient intensities above the background signal, is typi- 


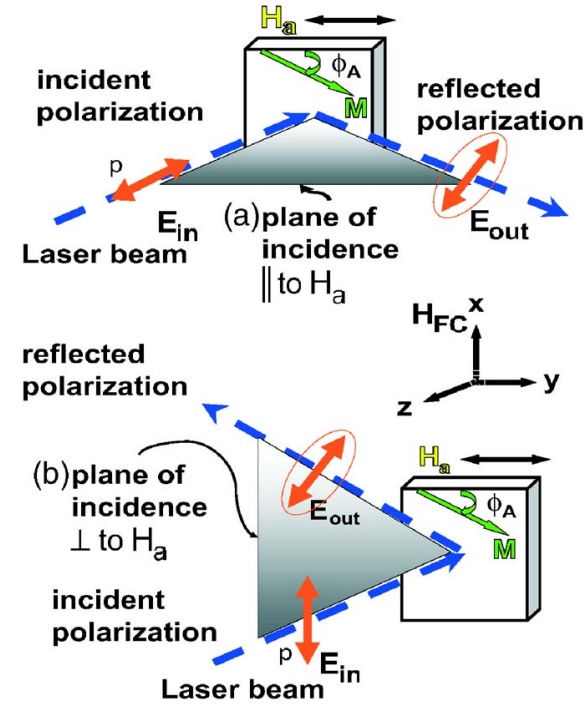

FIG. 1. (Color online) A schematic sketch of the MOKE experiment geometry showing the direction of the applied field $H_{a}$ with respect to the $H_{\mathrm{FC}}$ direction and the corresponding in-plane mean magnetization direction $\phi_{A}$ for (a) longitudinal and (b) transverse component measurements.

cally $\approx 60 \mathrm{mrad}$ (for the present specimen) and a typical $\Delta \alpha$ of around $0.5 \mathrm{mrad}$ can be specified by adjusting the two slits before the sample, thus the fraction $\Delta \alpha / \alpha$ becomes $\approx 0.008$. Therefore, a $Q$ resolution of $\Delta Q / Q \approx 1.0 \%$ was chosen, which is typical for such reflectivity measurements. This resolution could also be changed (to some extent) to higher or lower values depending upon the system under investigation.

\section{EXPERIMENTAL RESULTS}

\section{A. Longitudinal and transverse MOKE}

Typical experimental MOKE loops (symbols) recorded within the longitudinal and transversal geometries and for different orientations of the sample are shown together with calculated curves (lines) in Figs. 2 and 3 for the $N=10$ sample and in Figs. 4 and 5 for the $N=2$ sample. The saturation $p$ ellipticities for the $N=2$ and $N=10$ samples are found be $(1.16$ and 0.56$) \times 10^{-4} \mathrm{rad}$, respectively. In the case of $N=2,28 \%$ of the MOKE signal arise from the Permalloy buffer. Taking into account that the layers are thin and the multilayer consists of $1 / 3 \mathrm{Co}$ and $2 / 3$ nonferromagnetic IrMn the value of the ellipticity for $N=10$ is in basic quantitative agreement with the theoretical value of 1.54 $\times 10^{-4} \mathrm{rad}$, which we have calculated for a single thick Co film using the magneto-optic parameters from Ref. 15.

Considering the fact that the loops only reveal a significant contribution of the Permalloy buffer layer for $N=1$ and $N=2$, the MOKE can be estimated to be sensitive to roughly the two topmost bilayers, which is consistent with the limited information depth of the method. Thus, comparing the MOKE data of samples with increasing $N$ we can extract the variation of the magnetic parameters with $N$. From this analysis, we find that the magnetic parameters (exchange bias and uniaxial anisotropy strength) vary only slightly with $N$. For the theoretical description of the MOKE hysteresis loops it is therefore justified to take into account only one

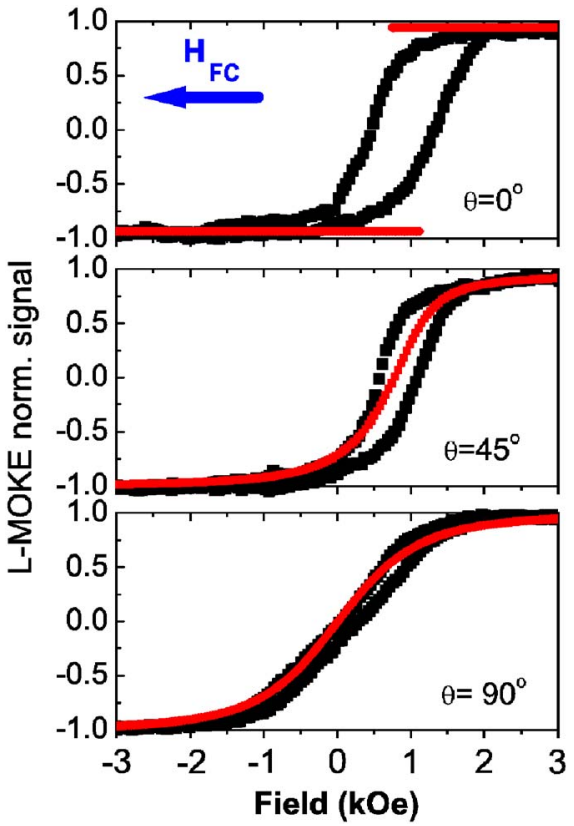

FIG. 2. (Color online) Longitudinal MOKE (L-MOKE) hysteresis loops for $\left[\mathrm{Ir}_{20} \mathrm{Mn}_{80}(6.0 \mathrm{~nm}) / \mathrm{Co}_{80} \mathrm{Fe}_{20}(3.0 \mathrm{~nm})\right]_{10} \mathrm{ML}$ when $\theta=0^{\circ}, \theta=45^{\circ}$, and $\theta$ $=90^{\circ}$. The calculated curves are also shown (lines).

effective Co layer and one Permalloy layer (for $N=1,2$, and $3)$. The calculation of the magnetization angle versus applied field has been performed employing a single domain model, taking into account the Zeeman energy due to the applied field, the exchange bias energy as an effective bulk effect, and a uniaxial anisotropy with the easy axis parallel to the exchange bias direction. As the Permalloy layer lacks a significant exchange bias and anisotropy, its magnetization has been assumed to be aligned in the field direction. The exchange bias strength has been determined from the shift of the easy axis loops $\left(\theta=0^{\circ}\right)$ and the uniaxial anisotropy strength by comparing the low field slope of the hard axis loops $\left(\theta=90^{\circ}\right)$ with the model calculation.

The MOKE ellipticity obtained from the longitudinal geometry has been modeled from the calculated magnetization angles taking into account only the first order terms that are linear in $\cos \phi_{A}{ }^{16}$ i.e.,

$$
\epsilon_{\text {long }}=\sum_{i}\left(l_{i} \cos \phi_{A, i}\right) .
$$

Here, $l_{i}$ are phenomenologically the first order MOKE coefficients, which have been extracted from the easy axis loops. The layer index $i$ is described either by the $\mathrm{NiFe}$ or the Co layer. On the other hand, the MOKE loops in the transversal geometry clearly show, in addition to the first order terms linear in $\sin \phi_{A}$, small but noticable second order effects $^{16}$ that are linear in $\cos \phi_{A}$. For $N \leqslant 3$, the second order terms, quadratic in $M$ are proportional to $\cos \phi_{A} \sin \phi_{A}$, that is,

$$
\epsilon_{\mathrm{trans}}=\sum_{i}\left(l_{i} \sin \phi_{A, i}+t_{i} \sin \phi_{A, i}+q_{i} \sin \phi_{A, i} \cos \phi_{A, i}\right) .
$$

Here $t_{i}$ are the coefficients with a value of $l_{i} / t_{i}=0.08$ and have been determined from the difference $\Delta \epsilon$ between + and 


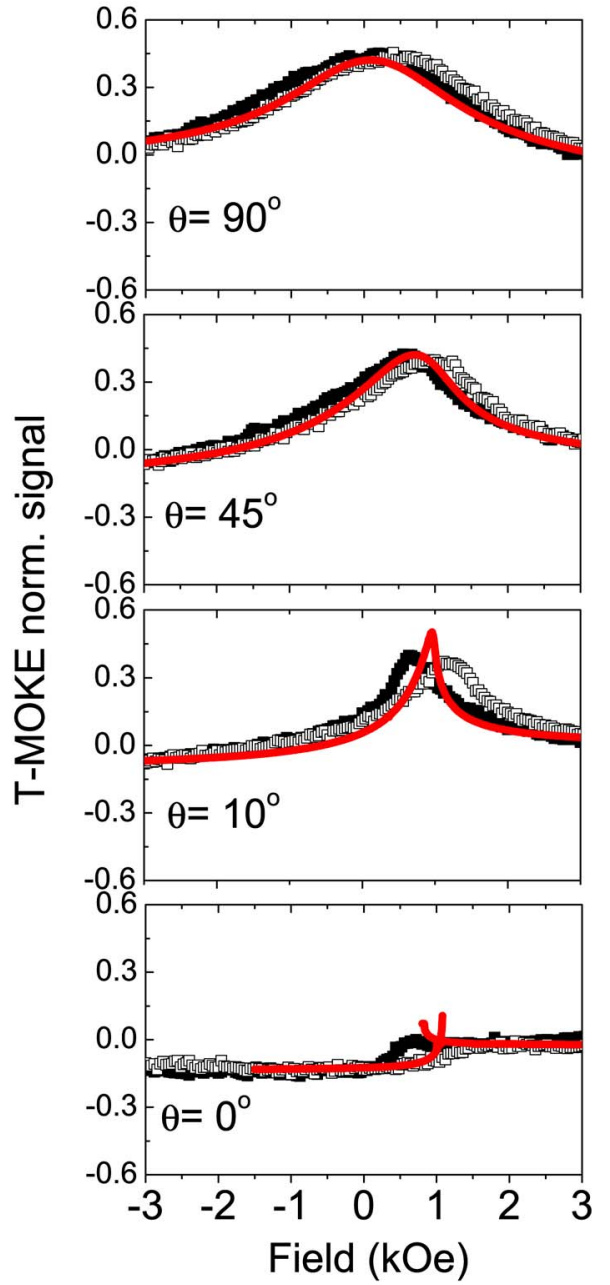

FIG. 3. (Color online) Transverse MOKE (T-MOKE) hysteresis loops for $\left[\mathrm{Ir}_{20} \mathrm{Mn}_{80}(6.0 \mathrm{~nm}) / \mathrm{Co}_{80} \mathrm{Fe}_{20}(3.0 \mathrm{~nm})\right]_{10} \mathrm{ML}$ when $\theta=0^{\circ}, \theta=10^{\circ}, \theta=45^{\circ}$, and $\theta=90^{\circ}$ along the decreasing (open square) and increasing branches (solid square). The calculated curves are also shown (lines).

- saturations of the transversal easy axis loops $\left(\theta=0^{\circ}\right)$. While the $q_{i}$ coefficients with a value of $q_{i} / l_{i}=0.18$ have been determined from the asymmetry of the transversal hard axis loops $\left(\theta=90^{\circ}\right)$, the calculated MOKE loops are in excellent agreement with the experimental data, except for the field range, where a switching via domain formation can be expected. This agreement is astonishing; as with the polycrystalline nature of the samples also some degree of spin disorder can be expected to arise due the magnetocrystalline anisotropy and the random grain orientations.

\section{B. Neutron scattering}

\section{Neutron specular scattering}

We performed PNR measurements with polarization analysis on both sides of the hysteresis loop at 15 different field values, with the field applied at different angles to the $H_{\mathrm{FC}}$ direction. A scheme of the scattering geometry with respect to $\phi_{A}$, the angular variation of magnetization, is shown in Fig. 6 for $\theta=90^{\circ}$ with respect to the $H_{\mathrm{FC}}$ direction. We show the measurements at $\theta=90^{\circ}$ for increasing and decreasing branches of fields with respect to the $H_{\mathrm{FC}}$ direction in Fig. 7. Measurements at $\theta=45^{\circ}$ have been already reported

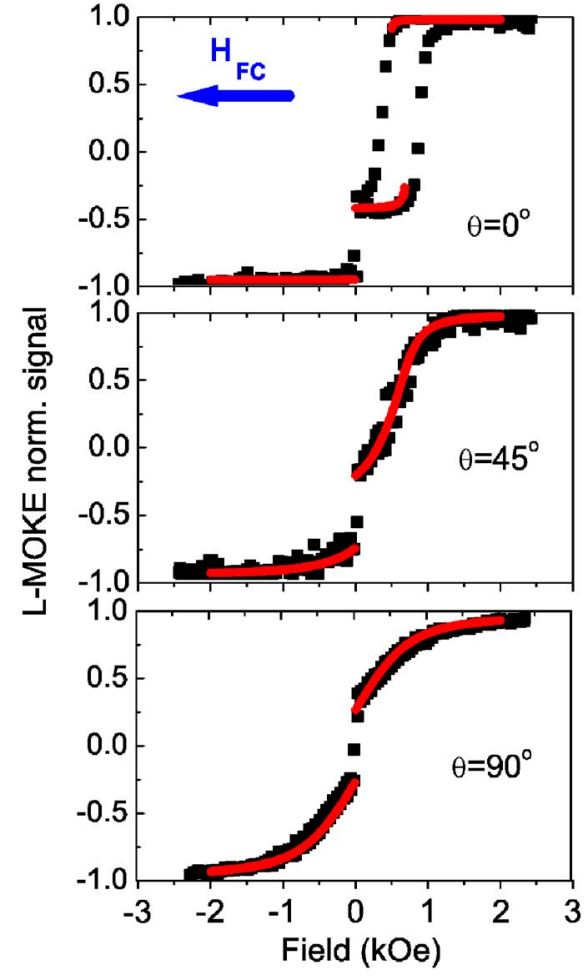

FIG. 4. (Color online) Longitudinal MOKE (L-MOKE) hysteresis loops for $\left[\mathrm{Ir}_{20} \mathrm{Mn}_{80}(6.0 \mathrm{~nm}) / \mathrm{Co}_{80} \mathrm{Fe}_{20}(3.0 \mathrm{~nm})\right]_{2} \mathrm{ML}$ when $\theta=0^{\circ}, \theta=45^{\circ}$, and $\theta$ $=90^{\circ}$. The calculated curves are also shown (lines). The effect of the buffer $\mathrm{NiFe}$ layer (showing no exchange bias effect) is visible and has been taken into account in the calculation.

previously. ${ }^{11}$ The specular intensity along the line $\alpha_{i}=\alpha_{f}$ shows first order and weaker second order Bragg peaks at $\alpha_{i, f} \approx 25$ and $50 \mathrm{mrad}$ corresponding to the bilayer thickness. The fits of the specular reflectivities for the NSF and SF channels are carried out, taking into account the nonideal polarization efficiencies. We find a reasonable agreement with the nominal values of film thicknesses and the nuclear and magnetic scattering length densities without assuming any interfacial layer between the AF and FM. The details of the fitting procedure have been described earlier. ${ }^{8}$ In Fig. 8 we show $\phi_{A}$ extracted from the fits to the respective specular scattering curves (reconstructed from Ref. 11). When the magnetization is parallel to the applied field $\phi_{A}$ should be $=0^{\circ}$. Thus, the increase in $\phi_{A}$ is extracted directly from the increase in the SF signal. For $\theta=45^{\circ}$ and $90^{\circ}$, we observe a large specular SF signal all along the hysteresis loop, indicating a uniform magnetization reversal. The fits to the curves are done considering all the layers to rotate coherently with the applied field. We do not observe any variation in the width of the Bragg peak for our whole set of data, which again indicates an apparently coherent behavior along the ML stack. The variation is monotonic and gradually follows the $H_{a}$ direction with increasing field strengths. The hysteresis observed from the fits is consistent with the hysteresis observed in the magnetization measurements in Fig. 2.

\section{Neutron off-specular scattering}

Figure 9 shows the SF intensity $\left(R_{+-}\right)$maps as a function of $\alpha_{i}$ and $\alpha_{f}$ at different representative $H_{a}$ for the angles $\theta$ 


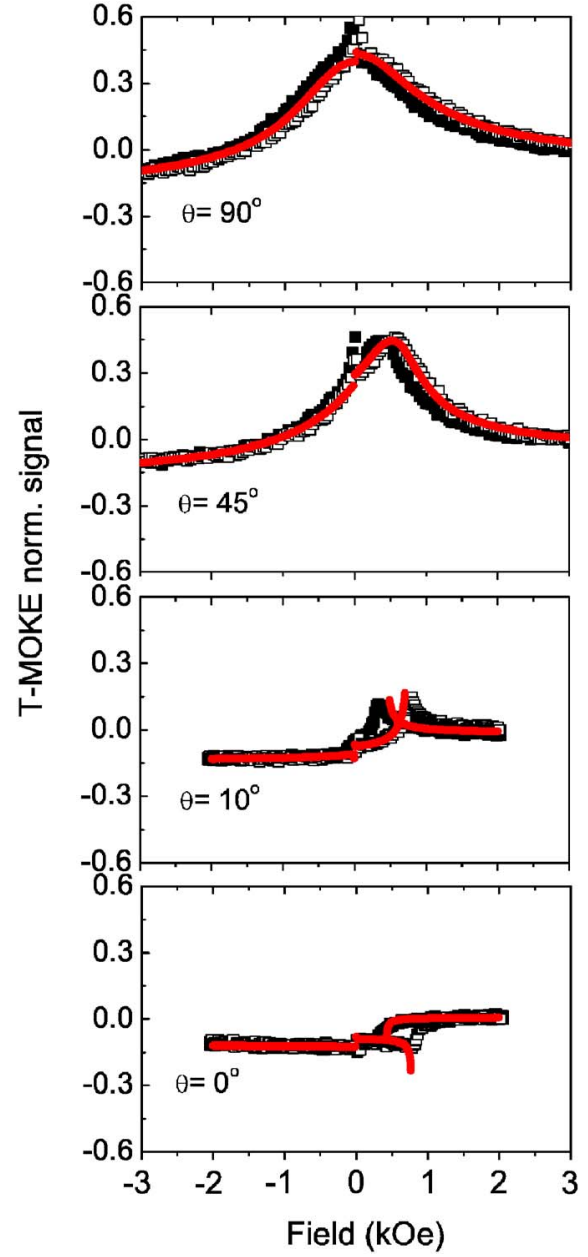

FIG. 5. (Color online) Transverse MOKE (T-MOKE) hysteresis loops for $\left[\mathrm{Ir}_{20} \mathrm{Mn}_{80}(6.0 \mathrm{~nm}) / \mathrm{Co}_{80} \mathrm{Fe}_{20}(3.0 \mathrm{~nm})\right]_{2} \mathrm{ML}$ when $\theta=0^{\circ}, \theta=10^{\circ}, \theta=45^{\circ}$, and $\theta=90^{\circ}$ along the decreasing (open square) and increasing branches (solid square). The calculated curves are also shown (lines). Here also the effect of the buffer NiFe layer is taken into account.

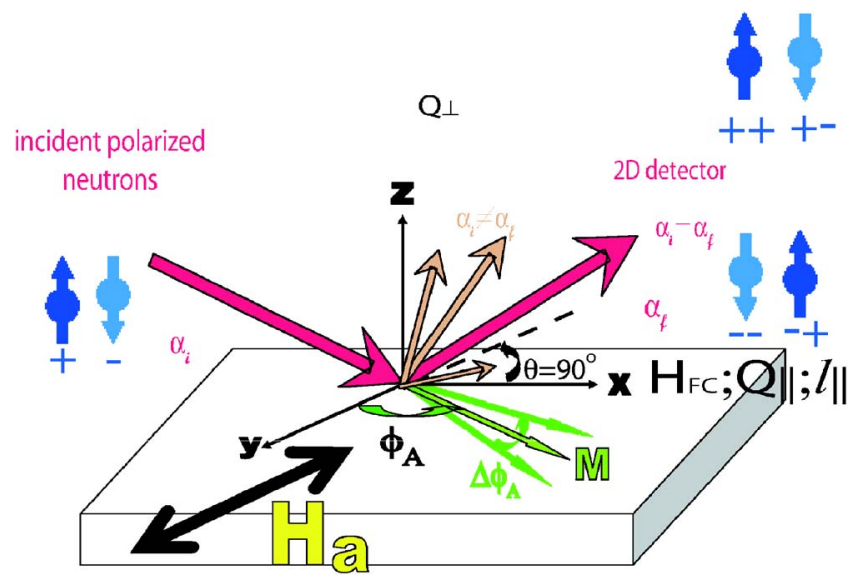

FIG. 6. (Color online) A schematic sketch of the neutron scattering geometry showing the direction of the applied field $H_{a}$ for $\theta=90^{\circ}$ with respect to the $H_{\mathrm{FC}}$ direction and the corresponding in-plane mean magnetization direction $\phi_{A}$ along with the random fluctuation $\Delta \phi_{A}$ around the direction of mean magnetization. Four combination of the two spin states can be achieved by two flippers (one before the sample in front of the polarizer and the other before the analyzer and after the sample) to yield the respective specular $\left(\alpha_{i}=\alpha_{f}\right)$ and off-specular $\left(\alpha_{i} \neq \alpha_{f}\right)$ intensities in the NSF and SF channels. $=45^{\circ}$ and $90^{\circ}$ (represented from Ref. 11). We also present here similar SF maps for $\theta=15^{\circ}$ and $0^{\circ}$, for comparison. The SF maps are plotted for a comparable level of the offspectacular intensities, as they have been recorded during different periods of time corresponding to different reactor periods. An enhanced off-spectacular intensity in the SF channel appears near the critical angle $\left(\alpha_{c} \approx 4 \mathrm{mrad}\right)$ of total reflection for the reversal points as compared to that in the saturation state. The sizable values of the off-specular SF intensity confirms that the in-plane magnetization component perpendicular to the guiding field $M_{\perp}$ is laterally inhomogeneous on a length scale smaller than the lateral coherence length of the neutron beam.

\section{DISCUSSION}

\section{A. Theoretical model for the reversal mechanism in an exchange bias system}

We explain the above observation of different magnetization reversals for different $\theta$ by considering a simple model where the relevant energy terms per unit volume can be written as

$$
\begin{aligned}
\frac{E}{M_{\mathrm{FM}}} & =-H_{a} \cos (\delta-\theta)-H_{X} \cos \delta+\frac{K}{M_{\mathrm{FM}}} \sin ^{2} \delta \\
& =H_{a}(\delta, \theta)+H_{X}(\delta)+H_{A}(\delta) .
\end{aligned}
$$

Here, $J$ is the interlayer exchange strength between FM and $\mathrm{AF}$ layers and $\delta$ is the angle between the $M_{\mathrm{FM}}$ and the easy axis. Also $H_{X}=J M_{\mathrm{AF}}$ as $M_{\mathrm{FM}}$ and $M_{\mathrm{AF}}$ (uncompensated spins) are the saturation magnetization of the FM and magnetization of the AF layer, respectively, and $K$ is the uniaxial anisotropy of the FM. Here we consider $M_{\mathrm{AF}}$ along the easy axis, which is assumed to be parallel to the $H_{\mathrm{FC}}$ direction. We furthermore assume that $M_{\mathrm{AF}}$ does not rotate with the $H_{a}$ direction. ${ }^{19}$ For a finite $\theta$, the strength of the anisotropy field $H_{A}$ (depends on the projection of the FM magnetization onto the easy axis) and that of the exchange field of the AF decide on the angle taken between the effective field $H_{\text {eff }}\left(H_{\text {eff }}\right.$ $\left.=H_{a}+H_{A}+H_{X}\right)$ and the $M_{\mathrm{FM}}$ direction $\left(\phi_{1}\right)$ at equilibrium for the increasing branch of the hysteresis loop. As the sign of FM magnetization (decreasing branch of the hysteresis loop) reverses, so does the anisotropy field and the corresponding angle $\phi_{2}$ can be very close to $180^{\circ}$. $^{7,9}$ A larger angle corresponds to a larger torque, which favors the rotation of the magnetization, whereas a small angle favors flipping by domain nucleation and wall motion. A sketch showing the angle, which $H_{\text {eff }}$ makes with the $M_{\mathrm{FM}}$ for representative strengths of the anisotropy and exchange field, is given in Fig. 10. When $\theta$ is large $\left(\theta=45^{\circ} / 90^{\circ}\right)$ and $H_{X}$ is larger than $H_{A}, H_{\text {eff }}$ makes a large angle $\left(\phi_{1} \approx \theta\right)$, favorable for coherent rotation for both branches [see Fig. 10(a)]. ${ }^{11}$ However, when $H_{X} \simeq H_{A}$ an asymmetric reversal can be seen in Monte Carlo simulations. ${ }^{7}$ We also observed this behavior experimentally on our ML specimens ${ }^{9,10}$ with the flipping of the magnetization only along the increasing branch $\left(\phi_{1} \approx 0^{\circ}\right)$ of the hysteresis loop. On the other hand, for $\theta$ very close to zero, the magnetization angle $(\delta)$ is also very small and thus $\phi$ is always nearly zero favoring only flipping [Fig. 10(b)]. ${ }^{8,9}$ 

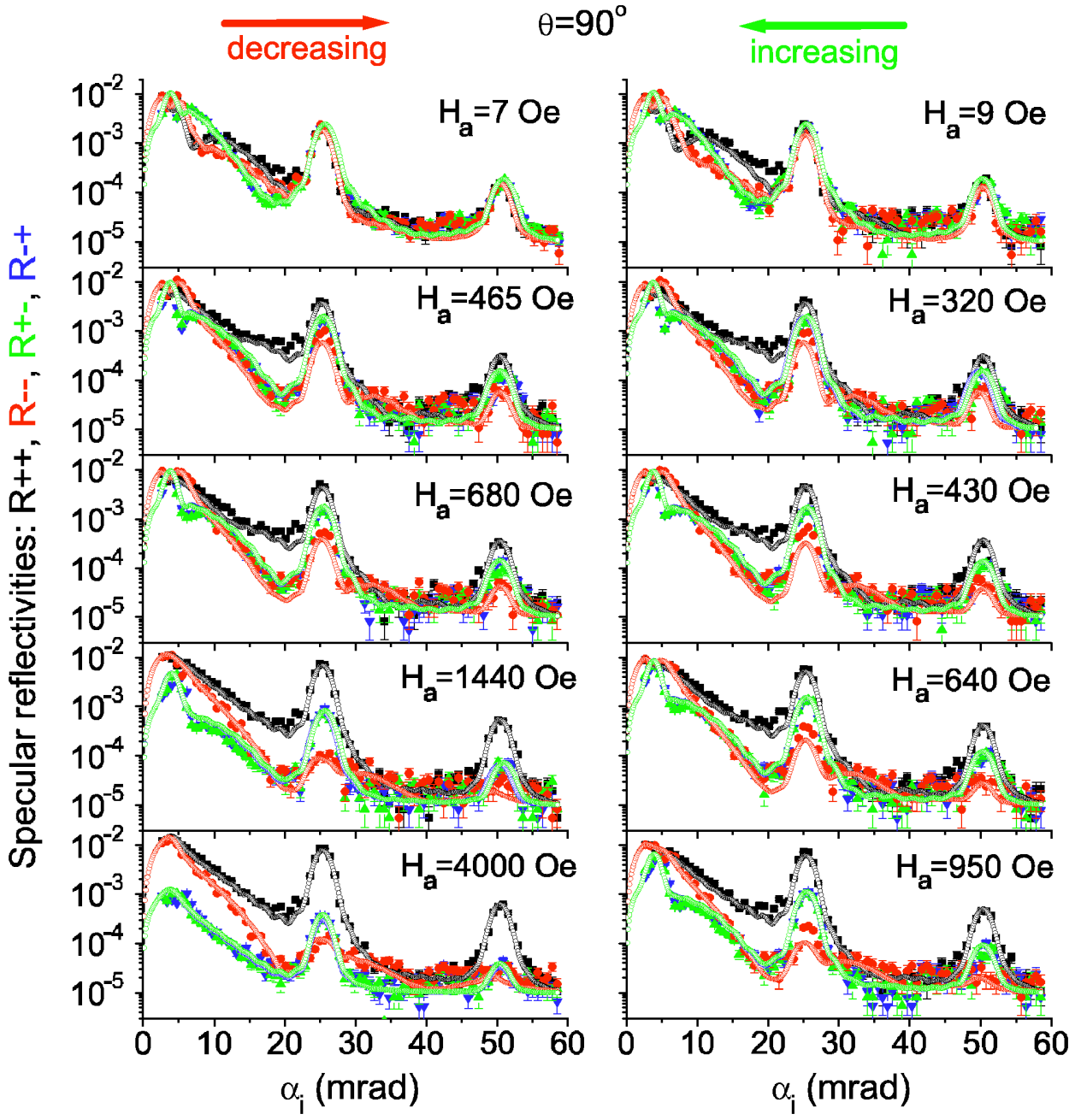

FIG. 7. (Color online) Specular reflectivity patterns of the NSF $\left[R_{--}\right.$(red/ dark gray circle) and $R_{++}$(black square) $]$ and SF channels $\left[R_{+-}\right.$(green/ light gray triangle) and $R_{-+}$(blue/black downtriangle)] along with their fits (open circle) for $\left[\mathrm{Ir}_{20} \mathrm{Mn}_{80}(6.0 \mathrm{~nm}) /\right.$ $\left.\mathrm{Co}_{80} \mathrm{Fe}_{20}(3.0 \mathrm{~nm})\right]_{10}$ ML when $\theta=90^{\circ}$ for decreasing and increasing branches of fields.

\section{B. Longitudinal and transverse MOKE}

Recently, the role of the anisotropy energy ratio $K / J_{X}$ has been highlighted by Camarero et al. ${ }^{17}$ and Paul et al., ${ }^{10}$ with $J_{X}$ and $K$ being the undirectional and uniaxial anisotropies of the FM layer, respectively. In our case, the exchange

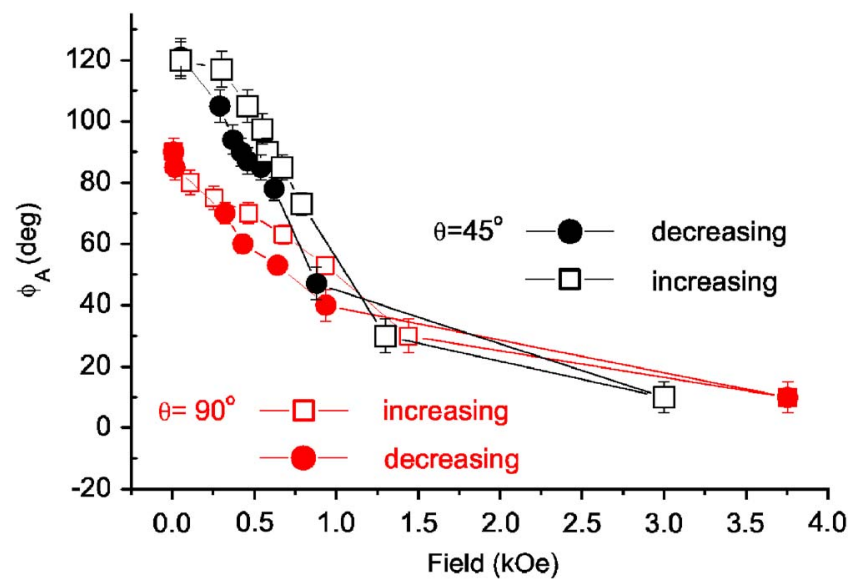

FIG. 8. (Color online) Angular variation $\phi_{A}$ of the magnetization vector for $\left[\mathrm{Ir}_{20} \mathrm{Mn}_{80}(6.0 \mathrm{~nm}) / \mathrm{Co}_{80} \mathrm{Fe}_{20}(3.0 \mathrm{~nm})\right]_{10} \mathrm{ML}$ when $\theta=45^{\circ}$ (black) and $90^{\circ}$ (red/dark gray) for increasing (open squares) and decreasing (closed circles) branches of fields as resolved from the fits to the specular reflectivity patterns. The uncertainty in determining the angles is $\pm 5^{\circ}$. The lines are guides to the eye. anisotropy energy is given by $J_{X}(N)=M_{\mathrm{FM}} H_{X}$ and can be estimated from the longitudinal MOKE loops from samples of the same layer thicknesses but of various $N$. Additionally, we also find a variation in the $K$ values with $N$. The uniaxial anisotropy energy is thus given by $K(N)=\left(H_{s} M_{\mathrm{FM}}\right) / 2$ and this can be estimated from the fits to the hysteresis loops of samples with different $N$ measured at $\theta=90^{\circ}$ [done by energy minimization of Eq. (4)]. Given the fact that our single multilayered system has different strengths of the exchange bias fields $\left(H_{X}\right)$ along the ML stack, this should also result in different ratios of $K / J_{X} \cdot{ }^{10}$

For $\theta=0^{\circ}$, the reversal mechanism is fairly symmetric (irrespectively of $N$ ) for both loop branches and is via domain nucleation and wall motion. This is in agreement with the findings from the neutron reflectivity for $N=3$ and $N$ $=10$ MLs presented earlier in Refs. 8 and 12. In the present case, for a slightly higher angle, e.g., $\theta=10^{\circ}$, one can see a small asymmetry in the decreasing and increasing branches even from the MOKE curves-although the overall reversal mechanism is predominantly rotational. Such an asymmetry almost disappears for higher values of $\theta$.

In Fig. 11 we show the angular variation $\phi_{A}$ of the magnetization magnetization for $N$ layers in the $N=10 \mathrm{ML}$ specimen for $\theta=90^{\circ}$ at $H_{a}=1440$ Oe along the decreasing branch of the hysteresis loop, which has been extracted from the MOKE curves with varying $N$. For an angle $\theta \approx 10^{\circ}-15^{\circ}$, 


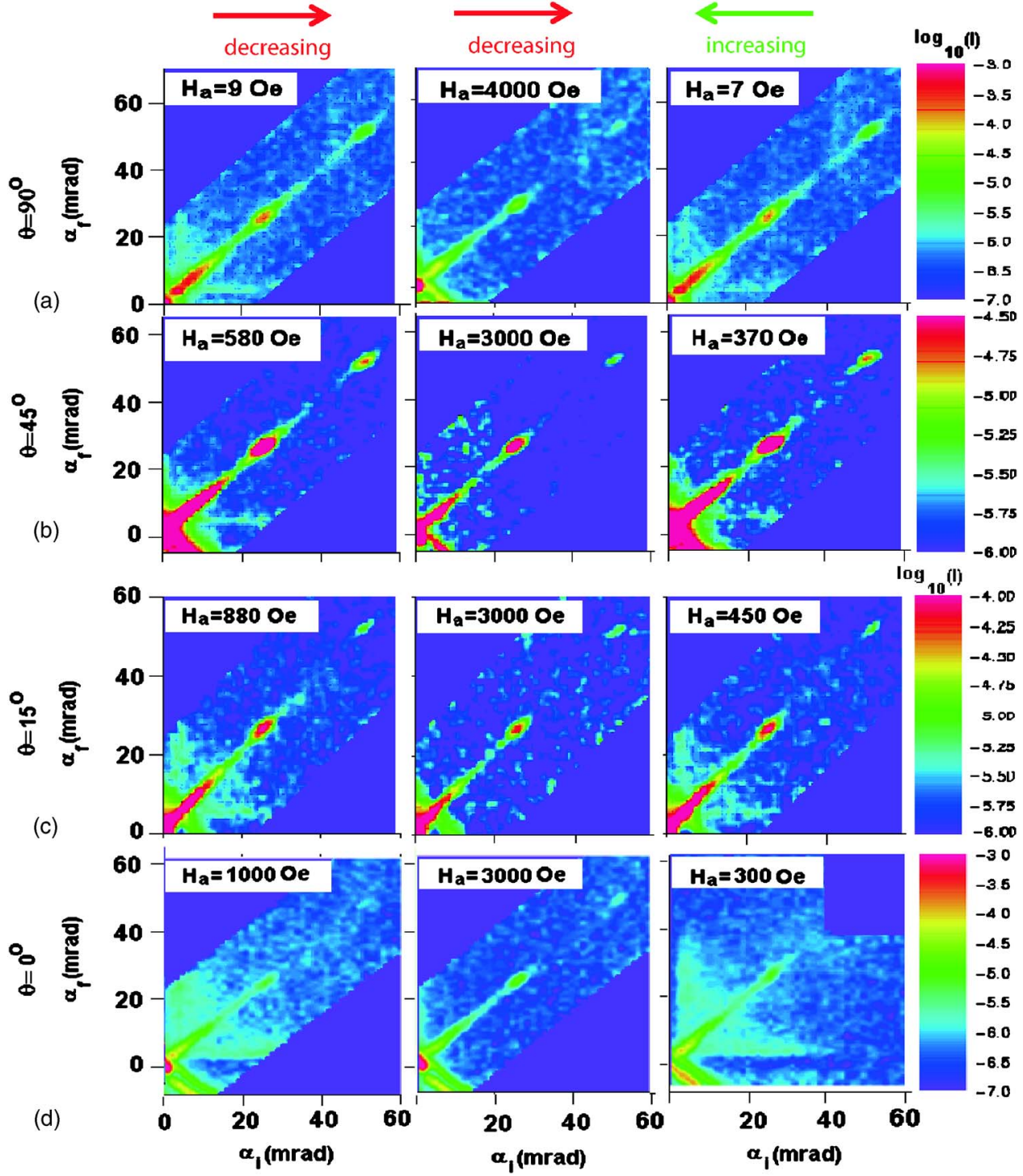

FIG. 9. (Color online) SF intensity maps $\left(R_{+-}\right)$for $\left[\mathrm{Ir}_{20} \mathrm{Mn}_{80}(6.0 \mathrm{~nm}) /\right.$ $\left.\mathrm{Co}_{80} \mathrm{Fe}_{20}(3.0 \mathrm{~nm})\right]_{10} \mathrm{ML}$ at the respective reversal points: (a) 9 Oe (decreasing branch) and $7 \mathrm{Oe}$ (increasing branch) when $\theta=90^{\circ}$ and followed by (b) 580 Oe (decreasing branch) and 370 Oe (increasing branch) when $\theta$ $=45^{\circ}$ [the maps in (a) and (b) are represented from Ref. 11]. We show, for comparison, the respective reversal points: (c) 880 Oe (decreasing branch) and 450 Oe (increasing branch) when $\theta=15^{\circ}$ and followed by (d) $1000 \mathrm{Oe}$ (decreasing branch) and 300 Oe (increasing branch) when $\theta=0^{\circ}$ [the map in (d) is represented from Ref. 12]. the extraction of the angles from individual layers becomes difficult as we may have a comparable signal out of the domain nucleation as well as the rotational reversal. In this case, the depth sensitivity of (chemical/nuclear and magnetic) polarized neutron reflectivity can be more helpful. ${ }^{10}$

\section{Neutron scattering}

\section{Neutron specular scattering}

One may note from Fig. 8, that for $\theta=45^{\circ}$, when we are very close to the remanent field $\left(H_{a} \approx 0\right)$, the angle $\left(\phi_{A}\right)$ is only $120^{\circ}$ and not $135^{\circ}$. The reason for this is simply the minimum magnetic field that is needed to measure the neutron results, which is $\approx 50$ Oe. Such small fields at the sample position are necessary in order to maintain the polarization of the neutron beam. However, for $\theta=90^{\circ}$ the actual field for the measurement was set to a lower value, much closer to zero $(\approx 10 \mathrm{Oe})$, and thus the value for $\phi_{A}$ is also found to be close to $90^{\circ}$. At these states of remanence the amount of rotation that each layer undergoes is fairly similar for increasing and decreasing field sweeps. This behavior is in agreement with the situation discussed by Camarero et $a l .{ }^{17}$ by using MOKE: Small differences in the $M_{\perp}$ compo- nent for increasing and decreasing branches have been observed for $\theta$ close to $0^{\circ}$ (e.g., $\pm 9^{\circ}$ ), which of course disappear at higher angles (e.g., $\pm 45^{\circ} / 90^{\circ}$ ). This again is primarily dependent upon the anisotropy ratios in respective systems. In our case, we do observe some differences in the angular variation for the two branches, which can be owed to the depth sensitivity of the neutron technique compared to the Kerr one. Nevertheless, it is worthwhile to mention that due to the difference in exchange and anisotropy fields in each layer along the stack of our ML an exact agreement with a bilayer system should not be expected.

Further, the samples of Camarero et al. ${ }^{17}$ show exchange fields of around $150 \mathrm{Oe}$, which is consistent with the thickness of the FM layer (two times the thickness of our specimens). The range of exchange fields is comparatively higher in our case. It ranges from around $300 \mathrm{Oe}$ at the bottom layer to $900 \mathrm{Oe}$ at the top layer of the ML. This variation of exchange fields may be the reason why we do not observe an asymmetric reversal as predicted by Beckmann et al. ${ }^{7}$ for $\theta$ $=60^{\circ}$. A significant difference in the magnetization rotation for the increasing and decreasing branches was predicted for $\theta=72^{\circ}$ due to a change in sign of the anisotropy ratio, but 

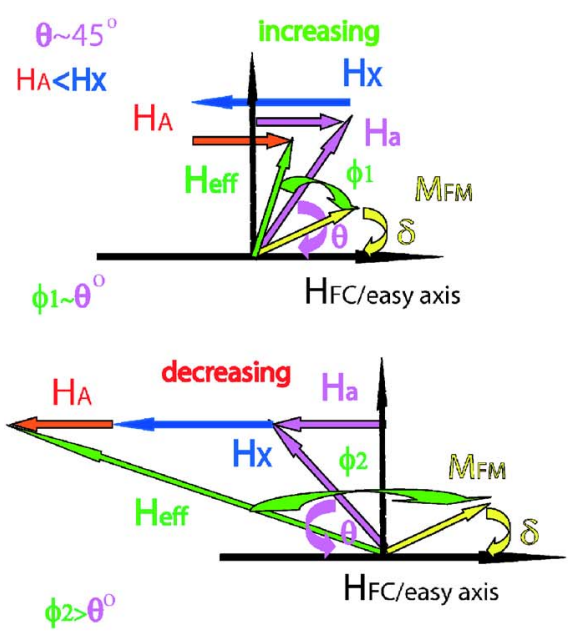

(a) Uniform reversal (rotation)

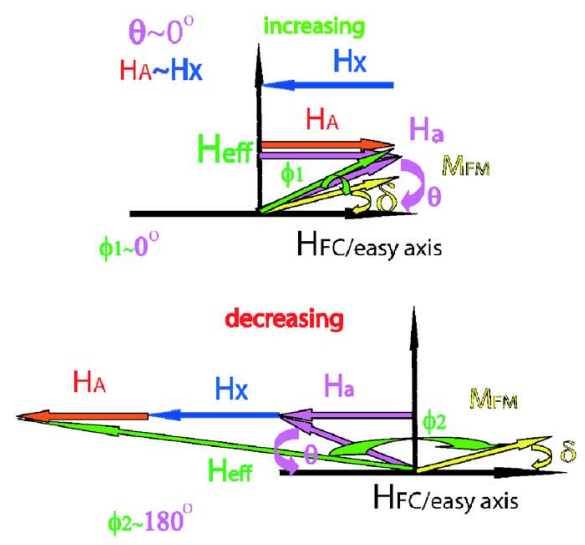

(b) Non-uniform reversal (domain wall)

FIG. 10. (Color online) Sketch showing the $H_{\text {eff }}$ (green/light gray) when (a) $\theta \approx 45^{\circ}$ for $H_{X}$ blue/black $\gg H_{A}$ (red/dark gray) undergoing uniform reversal. When $\theta \approx 0^{\circ}$, situations as (b) $H_{X} \approx H_{A}$ are shown for nonuniform reversal. The $M_{\mathrm{FM}}$ (yellow/light gray) and $H_{a}$ (violet/gray) are making angles $\delta$ and $\theta$, respectively, with the easy axis which is the $H_{\mathrm{FC}}$ direction. The components of the fields $\perp$ to $H_{\mathrm{FC}}$ has been exaggerated.

such a change is not expected to occur in our case. Thus, the small differences that we see at somewhat higher fields (above $0.5 \mathrm{kOe}$ in Fig. 8) are owed to the changes in the anisotropy ratio without any change in sign.

It may also be noted that the saturating field for the case when $\theta=0^{\circ}$ was $\approx 4.0 \mathrm{kOe},{ }^{8}$ as determined from specular

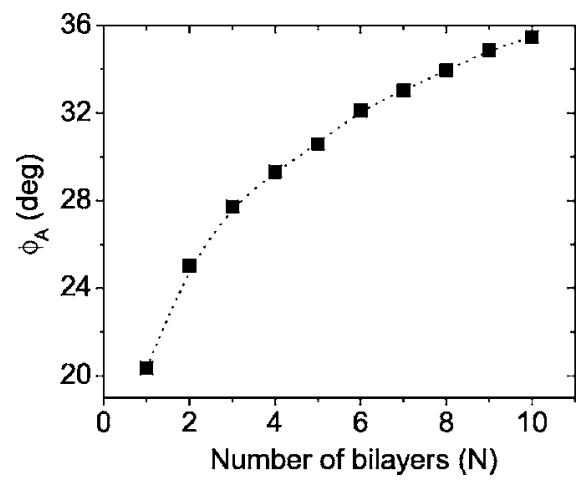

FIG. 11. Angular variation $\phi_{A}$ of the magnetization of $N$ layers in $\left[\mathrm{Ir}_{20} \mathrm{Mn}_{80}(6.0 \mathrm{~nm}) / \mathrm{Co}_{80} \mathrm{Fe}_{20}(3.0 \mathrm{~nm})\right]_{10} \mathrm{ML}$ when $\theta=90^{\circ}$ at $H_{a}=1440 \mathrm{Oe}$ along the decreasing branch of the hysteresis loop as extracted from the fits to the MOKE curves. The lines are guides to the eye. neutron scattering as well as conventional magnetometry methods. However, the extrapolation of the MOKE results in the present case reveals that such a field is insufficient to obtain a similar state of complete saturation for $\theta=45^{\circ}$ or $90^{\circ}$, owing to the additional exchange anisotropy induced in the system. This is also in agreement with our specular neutron results, where we find that $\phi_{A}$ is not exactly equal to $0^{\circ}$, but takes a value of $\approx 10^{\circ} \pm 5^{\circ}$, if a comparable field is applied along the easy axis (see Fig. 8). For a case close to saturation, the magnitude of the magnetization from the NSF intensities is often similar to that found for perfect alignment. A small deviation of the layer magnetizations from the collinear alignment along the applied field direction $\left(\phi_{A}\right.$ $=0^{\circ}$ ) does not make any significant difference in the magnitude of the parallel component (NSF intensities) compared to the perfect alignment: $M_{\|} / M_{\mathrm{FM}} \propto\left\langle\cos \left(\phi_{A}\right)\right\rangle \approx\left\langle 1-\left(\phi_{A}\right)^{2} / 2\right\rangle$. Whereas SF scattering is sensitive to the components perpendicular to $H_{a}, M_{\perp} / M_{\mathrm{FM}} \propto\left\langle\sin ^{2}\left(\phi_{A}\right)\right\rangle \approx \phi_{A}^{2}$. Now, when $\phi_{A}$ is small, then the difference in the NSF specular signal becomes insignificant. Earlier, in the case of AF coupled $\mathrm{Co} / \mathrm{Cu}$ layers measured along the hard axis, we have shown the true saturation field to be much higher (estimated from the off-specular neutron scattering from AF coupled domains) than predicted by conventional magnetometry methods. ${ }^{18}$ Similar off-specular scattering, however, is not expected to appear in the present case, and thus we cannot estimate the true saturation field along the hard axis from neutron scattering only.

\section{Neutron off-specular scattering}

Off-specular SF scattering shows that the coherent rotation of layers is accompanied by underlying concomitant inplane magnetization fluctuations. At saturation the diffused intensities disappear, which confirms their purely magnetic origin. These fluctuations-linked to the magnetization reversal - are due to the lateral random variation of the magnetization direction $\left(\Delta \phi_{A}\right)$ from domain to domain around their mean value. The lateral correlation length for such fluctuations has been estimated earlier from the simulation of the SF intensity maps under the distorted wave born approximation. ${ }^{12}$ The reference Hamiltonian is obtained from the fit to the specular reflectivity. The residual potential includes the fluctuations. Such small scale fluctuations, which are comparable to the grain size $(<1 \mu \mathrm{m})$, have been also observed earlier in case of $\theta=0^{\circ}$ (Ref. 8) and also $\theta=15^{\circ}$ (Ref. 10). They are observed, even when $\theta=45^{\circ}$ and $90^{\circ}$, where reversal takes place by coherent rotation only, thus indicating that these small scale fluctuations from domain to domain are smaller than the lateral projection of the neutron coherence length. Such off-specular scattering may vanish when the size of the domains becomes larger than the coherence length, such as in Ref. 9. However, the intensities for $\theta=15^{\circ}$ and $0^{\circ}$ are larger compared to those for higher angle measurements, thus indicating the decreasing degree of instability in the system with $\theta$ (Fig. 9). Moreover, the diffuse scattering intensities are observed along the Yoneda ridges only-and not at the Bragg peak position. This means that the fluctuations are incoherent through the stack of the ML, 


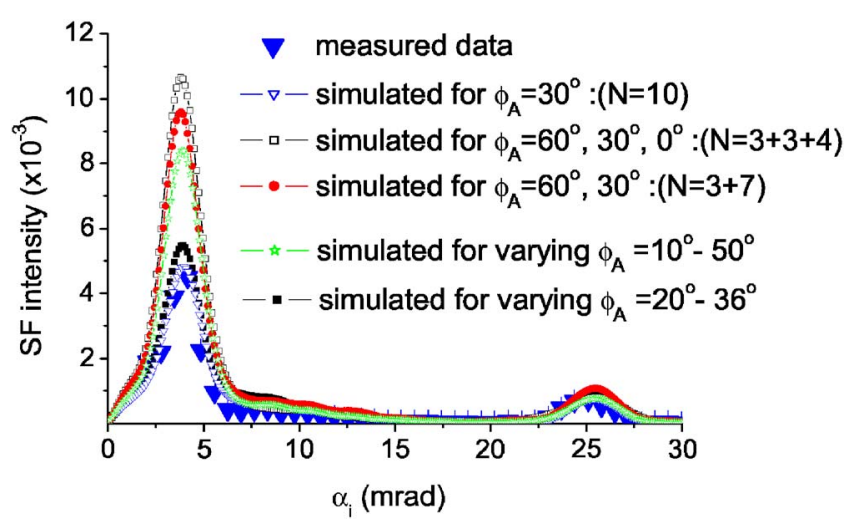

FIG. 12. (Color online) We compare the SF intensities for the ML considering different options of layer orientation, i.e., different $\phi_{A}$, depicting the situation at $H_{a}=1440$ Oe along the decreasing branch for the case where $H$ is at $90^{\circ}$ to the $H_{\mathrm{FC}}$ direction. Note that the differences in the intensity around the critical angle of the total reflection are justifying the assumption of a simultaneous rotation of all layers as observed from the neutron data.

which can be related to domains mediated by variable grain sizes through the ML stack.

\section{Complimentary information from PNR and MOKE}

Taking into account the differences in exchange energies for adjacent layers in the ML stack, one would expect a torque acting on each of the FM layers, the strength of which varies from layer to layer. In this case, one would expect that the angle by which each individual FM layer rotates at a certain field during the reversal is slightly different. However, we find that essentially all layer magnetizations are rotating simultaneously into the same direction.

With PNR being depth sensitive, one can determine the exact angle of each individual layer in a multilayer stack from the fits to the specular SF intensities with a great deal of accuracy. In the following, we want to clear any ambiguity regarding the level of accuracy of our assertion regarding a simultaneous rotation of all layers. For this purpose, we compare the experimental data to simulations, assuming different configurations for the magnetization structure in the multilayer. In Fig. 12, we show the comparison of the SF intensities (plotted on a linear scale instead of a log scale to emphasize the differences below $30 \mathrm{mrad}$, where the difference is clearly distinguishable) from the $N=10$ multilayer stack. The simulations are plotted for a field of $H_{a}$ $=1440$ Oe along the decreasing branch, when $\theta=90^{\circ}$. Five different configurations have been considered: (i) All layers are rotating by the same angle $\phi_{A}=30^{\circ}$; (ii) only the top three layers are rotating by $\phi_{A}=60^{\circ}$ and the bottom 7 layers by $\phi_{A}=30^{\circ}$; (iii) the three top layers are rotating by $\phi_{A}$ $=60^{\circ}$, the next three layers by $\phi_{A}=30^{\circ}$, and the last four layers by $\phi_{A}=0^{\circ}$; (iv) each layer rotates by a different angle along the stack (arbitrarily chosen) ranging from $10^{\circ}$ to $50^{\circ}$ from the bottom to the top layer; (v) each layer rotates subsequently by a different angle along the stack depending upon the different exchange field strengths - assuming a linear variation from $20^{\circ}$ to $36^{\circ}$ from the bottom to the top layer. The angles for configuration $(v)$ are estimated from the MOKE loops presented in the MOKE section. The simulated curves show the differences in the intensity profile for the

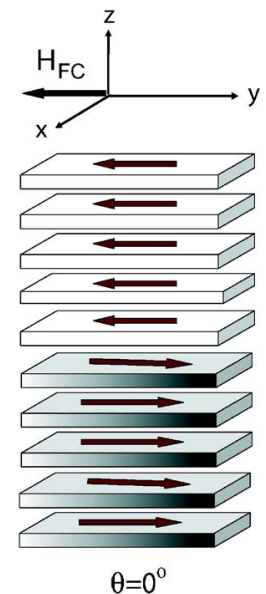

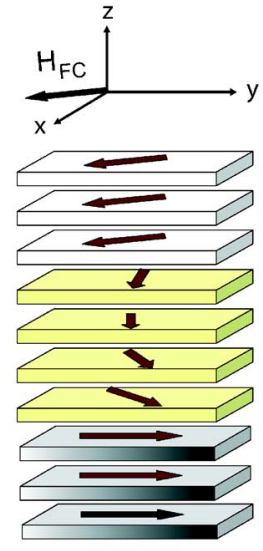

$\theta=15^{\circ}$

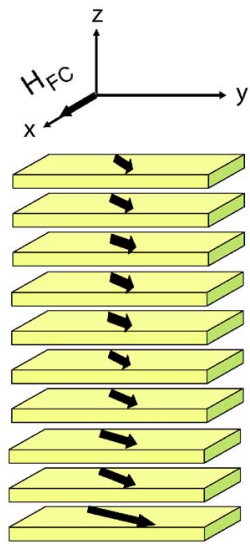

$\theta=90^{\circ}$
FIG. 13. (Color online) Schematic picture of the layer magnetization orientation for $N=10$ bilayer specimen along the decreasing branch when $\theta=0^{\circ}$ (Ref. 8) and $\theta=15^{\circ}$ (Ref. 10) (at $H_{a} \approx 1000$ Oe) as concluded from the PNR measurements. When $\theta=90^{\circ}$ (at $H_{a}=1440 \mathrm{Oe}$ ), the scheme is compatible with the MOKE results. $H_{\mathrm{FC}}$ makes an angle $\theta$ with respect to $H_{a}$ along the $y$ axis.

cases considered, justifying our conclusion of a similar orientation of all layer magnetizations drawn from the neutron specular data. The best fit to the measured results is obtained only when we consider the same angle of $\phi_{A}=30^{\circ}$ for all layers, i.e., configuration (i).

A coherent and simultaneous rotation of the magnetization in all layers of the multilayer stack explains the neutron scattering results. However, it partially disagrees with our MOKE results, which are more compatible with configuration (v). The question is thus how can the data consistently explain both configurations (i) and (v)? A solution may be found by looking into the properties of the two techniques in detail. Possibly, the magnitude of the transverse component obtained from the specular neutron data is less sensitive to a small variation $\Delta \phi$ of the rotation angle $\phi_{A}$ in each layer of the ML stack, whereas such information can be obtained for different multilayers with different $N$ by using MOKE. One may note that we have earlier estimated the mean magnetization direction for each individual layer of the same multilayer stack when $\theta=15^{\circ} .^{10}$ In this case, the angle over which the mean magnetization direction was seen to vary from the bottommost to the topmost layer was around $180^{\circ}$ ( $\phi_{A}$ varying from $0^{\circ}$ to $180^{\circ}$ ), compared to $\approx 16^{\circ}\left(\phi_{A}\right.$ varying from $20^{\circ}$ to $36^{\circ}$ ) in the present case. Figure 13 shows a schematic picture of the layer magnetization orientation for the $N=10$ bilayer specimen along the decreasing branch for $\theta=0^{\circ}$ (Ref. 8) and $\theta=15^{\circ}$ (Ref. 10) (at $H_{a} \approx 1000$ Oe) from the fits to the PNR curves. For $\theta=90^{\circ}$ (at $H_{a}=1440 \mathrm{Oe}$ ) the scheme is taken from the fits to the MOKE curves. The deviation from the mean direction being much smaller in the present case (e.g., $\theta=90^{\circ}$ ) becomes difficult to distinguish by PNR. The negative and positive magnetizations (due to change in sign of $\phi_{A}$ ) do not cancel out for $M_{\perp}$ components and are difficult to infer, but their differences do show up in $M_{\|}$components, making them far more easy to identify.

One may recall that the MOKE signal is limited only to the two topmost bilayers. The technique is based upon the magneto-optic effects that describe changes of the reflection 
coefficients from the magnetized media, which in the presence of a magnetic field (external/internal) can cause a change in the permittivity tensor of the material. PNR, on the other hand, measures the neutron scattering signal as the Fourier transform of the electron spatial distribution probed by the elastic magnetic scattering of the neutrons. Thus, the information that can be extracted for a multilayer stack largely depends upon the range of $Q$ vectors that can be probed (limited by the background intensity) with a suitable resolution and on the model that is invoked to fit the reflectivity profiles. The higher the number of layers in the multilayer, the larger is the number of fitting parameters involved (for nonperiodic magnetization arrangement along the stack of the ML) in determining the magnetization vector by neutron specular scattering. Moreover, the MOKE signal is obtained by averaging the magnetization direction over the whole area illuminated by the laser spot (large coherence length), whereas the average of the mean magnetization vector, as probed by neutrons, is the incoherent average over several coherence volumes $(\approx 5-10 \mu \mathrm{m})$. Due to these two different averaging procedures (affecting more the evaluation of the transverse component) over two different coherence volumes, a certain modulation, which can be observed in the MOKE signal, may be masked in PNR, as the changes within the specific coherence volumes (probed by neutrons) may not be similar.

\section{SUMMARY AND CONCLUSION}

We have studied in detail the remagnetization behavior of an exchange-biased ML, which was engineered to have an increasing exchange bias field with each subsequent layer along the stack. Thus, we intrinsically vary the relative strengths of the exchange and uniaxial anisotropies along the ML stack for a single multilayered system with $N$ bilayers. To gain an insight into the microscopic reversal mechanism, we varied the angle between the field cooling and applied field direction. This experimental work is thereby a typical example of cooling the system along a certain direction and measuring along various angles of applied fields with one easy axis.

By employing the neutron scattering technique (measuring NSF and SF scattering cross sections) for $N=10$ bilayer specimen, we find (i) a similar behavior for $\theta=45^{\circ}$ and $90^{\circ}$ cases, i.e., a uniform mode of magnetization reversal for a simultaneous rotation of all the layers on both remagnetization directions. This is different from the cases observed previously, for example, (ii) nonuniform and sequential switching of layers for $\theta=0^{\circ}$ and (iii) a layer dependent reversal by coherent rotation or domain nucleation and wall propagation for $\theta=15^{\circ}$. However, all reversal modes (independent of the angle of the field applied) are accompanied by small scale domain-to-domain fluctuations. The perpendicular component of the in-plane magnetization, which is on a length scale comparable to the grain size, is observed as a nonzero offspecular polarized neutron scattering signal.

We also employed the MOKE technique (measuring longitudinal and/or transverse components of the magnetization) for different directions of $\theta$ for specimens with different rep- etitions of bilayers $(N=2$ and 10) and could determine the magnetization behavior for the two topmost layer in each ML. This information from the $N$ th layer of different specimens was compared with the corresponding layer in our $N$ $=10 \mathrm{ML}$ stack, which has been investigated with neutrons.

All these observations by neutron scattering and MOKE techniques are understood in general terms of the variation of the anisotropy ratio. A large (favoring rotation)/small (favoring domain nucleation and wall motion or nucleation) torque exerted on the system is due to a large/small angle of the effective field strength with the magnetization axis acting on each layer.

The investigation by MOKE also revealed a small variation of the rotational angle of the average magnetization for each layer in the stack, for geometries with $\theta=45^{\circ}$ and $90^{\circ}$. Our PNR measurements, however, are not capable to detect such small variations and with this technique we only observe an apparent simultaneous rotation of all layers. Our results clearly demonstrate that neither MOKE nor PNR alone is able to unravel the detailed magnetization distribution in a multilayer stack, if the deviation from the mean direction is small. The combination of PNR and MOKE, however, gives a much more refined picture of the magnetization behavior and the reversal processes.

\section{ACKNOWLEDGMENTS}

We are thankful to D. E. Bürgler for useful discussions, U. Rüker for cooperating in HADAS, and E. Kentzinger for giving access to the PNR software.

${ }^{1}$ W. H. Meiklejohn and C. P. Bean, Phys. Rev. 102, 1413 (1956).

${ }^{2}$ V. I. Nikitenko, V. S. Gornakov, A. J. Shapiro, R. D. Shull, Kai Liu, S. M. Zhou, and C. L. Chien, Phys. Rev. Lett. 84, 765 (2000).

${ }^{3}$ W.-T. Lee, S. G. E. te Velthuis, G. P. Felcher, F. Klose, T. Gredig, and E. D. Dahlberg, Phys. Rev. B 65, 224417 (2002).

${ }^{4}$ M. R. Fitzsimmons, P. Yasher, C. Leighton, I. K. Schuller, J. Nogués, C. F. Majkrzak, and J. A. Dura, Phys. Rev. Lett. 84, 3986 (2000).

${ }^{5}$ M. Gierlings, M. J. Prandolini, H. Fritzsche, M. Gruyters, and D. Riegel, Phys. Rev. B 65, 092407 (2002).

${ }^{6}$ F. Radu, M. Etzkorn, R. Siebrecht, T. Schmitte, K. Westerholt, and H. Zabel, Phys. Rev. B 67, 134409 (2003).

${ }^{7}$ B. Beckmann, U. Nowak, and K. D. Usadel, Phys. Rev. Lett. 91, 187201 (2003).

${ }^{8}$ A. Paul, D. E. Bürgler, and P. Grünberg, J. Magn. Magn. Mater. 286, 216 (2005); A. Paul, E. Kentzinger, U. Rücker, D. E. Bürgler, and P. Grünberg, Phys. Rev. B 70, 224410 (2004).

${ }^{9}$ A. Paul, E. Kentzinger, U. Rücker, and Th. Brückel, Phys. Rev. B 73, 092410 (2006); J. Phys.: Condens. Matter 19, 86229 (2007).

${ }^{10}$ A. Paul, E. Kentzinger, U. Rücker, and Th. Brückel, Phys. Rev. B 74, 054424 (2006).

${ }^{11}$ A. Paul, E. Kentzinger, U. Rücker, and Th. Brückel, J. Phys.: Condens. Matter 18, L149 (2006).

${ }^{12}$ A. Paul, E. Kentzinger, U. Rücker, D. E. Bürgler, and P. Grünberg, Eur. Phys. J. B 45, 249 (2005).

${ }^{13}$ B. P. Toperverg, in Matter and Materials (Forschungszentrum Jülich, Germany, 2002), Vol. 12, p. 247.

${ }^{14}$ J. F. Anker and G. P. Felcher, J. Magn. Magn. Mater. 200, 698 (1999).

${ }^{15}$ G. S. Krinchik and V. A. Artemev, JETP Lett. 26, 1080 (1968).

${ }^{16}$ K. Postava, D. Hrabovsky, J. Pistora, A. R. Fert, S. Visnovsky, and T. Yamaguchi, J. Appl. Phys. 91, 7293 (2002).

${ }^{17}$ J. Camarero, J. Sort, A. Hoffmann, J. M. García-Martín, B. Dieny, R. Miranda, and J. Nogués, Phys. Rev. Lett. 95, 057204 (2005).

${ }^{18}$ A. Paul, E. Kentzinger, U. Rücker, D. E. Bürgler, and Th. Brückel, Phys. Rev. B 73, 094441 (2006).

${ }^{19}$ H. Ohldag, A. Scholl, F. Nolting, E. Arenholz, S. Maat, A. T. Young, M. Carey, and J. Stöhr, Phys. Rev. Lett. 91, 017203 (2003). 